\title{
An Online Teaching Design of Oral English against COVID-19: An "Ideological-and-Political-Theories-Education-in-All-Courses" Perspective
}

\author{
Jiejing Pan ${ }^{1}$ \\ ${ }^{1}$ School of Foreign Studies, Guangzhou University of Chinese Medicine, Guangzhou, China \\ Correspondence: Jiejing Pan, School of Foreign Studies, Guangzhou University of Chinese Medicine, \\ Guangzhou, China.
}

Received: October 25,2020

Accepted: August 25, 2021

Online Published: August 27, 2021

doi: 10.5539/elt.v14n9p39

URL: https://doi.org/10.5539/elt.v14n9p39

This paper is supported by the 2021 Social Science Project of Guangzhou University of Chinese Medicine: A Study of the Overseas Media-Constructed Image and Value of Chinese Medicine against COVID 19 (Grant No. 2021 SKZX11).

\begin{abstract}
Under the outbreak of the Coronavirus Disease 2019 (COVID-19) and the philosophy of "Ideological and Political Theories Education in all Courses (IPTEC)" by China's Ministry of Education, college curriculum reform has become a pressing issue in both form and content. Oral English course is characterized with flexible organization and a wide selection of teaching materials, thus closely related to the shaping of college students' values. An online teaching mode of oral English featuring "DingTalk + WeChat Group + FiF" is proposed after a mining of "ideological and political elements", with the sophomore oral English course of School of Foreign Studies, Guangzhou University of Chinese Medicine as a case. The highlights of this mode are as follows. First, all links of the teaching design are permeated with ideological and political elements, which realizes the blending of explicit and implicit educations. Second, it supports teacher-to-student and student-to-student voice interactions in a multi-party manner at any time. Third, a complex is created where one online classroom is systematically nested in another among the various platforms. Fourth, group and single games enrich the organization of the classroom. Fifth, it provides private and convenient classroom and homework management.
\end{abstract}

Keywords: COVID-19, Ideological and Political Theories Education in all Courses, oral English, online teaching

\section{Introduction}

Since the emergence of the Coronavirus Disease 2019 (hereinafter referred to as "COVID-19") in Wuhan in December 2019, the cumulative number of confirmed cases reported in China has kept on rising. In order to effectively curb the spread of the epidemic, the Ministry of Education required to postpone the opening of the spring semester (Note 1), and issued an initiative on January 29th that all schools across the country should continue teaching using online platforms despite the suspension of classes (Note 2). Online teaching, or distance education, refers to a way of have classes with the Internet as the medium, by virtue of videos, audios and other teaching software or platforms. Thanks to the universal Internet and the fact that it's free of the restriction of time and space, online teaching provides a solution as a response to the above-mentioned requirement during this special period. On the one hand, throughout the overall teaching process, the characteristics of traditional classrooms are retained in terms of self-study and homework after class, avoiding extra cognitive or psychological burden for students. On the other hand, online classrooms could enrich the channels for students to acquire knowledge and skills, and therefore arouse their enthusiasm and motivation in a novel learning environment to the maximum.

"Ideological and Political Theories Education in all Courses" (hereinafter referred to as "IPTEC") is a comprehensive educational philosophy promoted nationwide by the Ministry of Education since 2018. According to the basic requirements of IPTEC, "Education should center around moral development and the cultivation of people. Ideological and political work must run through the whole teaching process... so that a coordinated effect could be achieved between all the other courses and ideological and political theories courses" 
(Xi, 2016). Specifically, through deepening the reform of curriculum objective, content, structure and mode, the ideology- and politics-oriented education in political identity, national awareness, cultural confidence and personality development should be integrated with the inherent knowledge and skills of various courses, for the purpose of realizing an organic combination of explicit and implicit educations (Wang \& Shi, 2020: 52). Therefore, by no means is it groundless to reckon that this long-term educational concept and the outbreak of the epidemic have shed light upon the urgence of college curriculum reform in both form and content.

\section{Previous Studies and Research Niche}

The advanced search function of CNKI is used to collect current studies with both “课程思政 (IPTEC)” and “在 线教学 (online teaching)" as the theme, screening just 24 articles. What is worth noting is that they are all publications within the year 2020, during which the outbreak necessitates this special teaching approach. In the 24 results screened, a general differentiation can be made between theoretical discussions of online teaching from the perspective of IPTEC, and IPTEC-guided specific online teaching designs. The former involves 6 articles, the proportion of which is $25 \%$ of the total (Cui \& Che, 2020; Han et al., 2020); the latter can, according to the level on which the designed is conducted, be further divided into teaching mode explorations for a certain discipline (4 articles), a certain major (4 articles) as well as a certain course (10 articles), which accounts for $16.7 \%, 16.7 \%$ and $41.6 \%$, respectively, as shown in Figure 1.

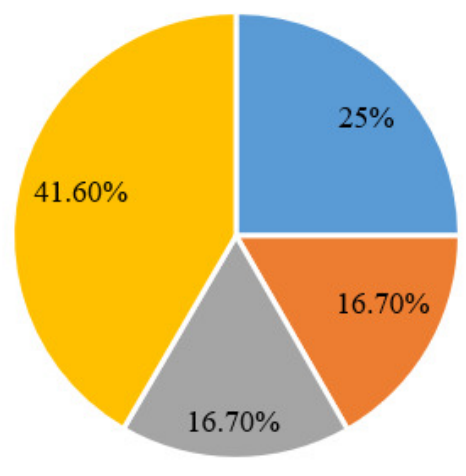

- General theoretical discussions

- Discipline-oriented teaching designs

- Major-oriented teaching designs

- Course-oriented teaching designs

Figure 1. Previous Studies on Online Teaching from a IPTEC Perspective

An in-depth examining of the 4 online teaching designs for a specific discipline singles out only 1 targeted at English language (Sun, 2020). Considering the degree of refinement, it is online teaching explorations for a certain course that are the most relevant to this study. The 10 course-oriented teaching designs mainly cover such classes as Immunology (Fu et al., 2020; Jiang et al., 2020), Pharmaceutical Analysis (Jin et al., 2020), Nursing (Xu et al., 2020), and Introduction to Philosophy ( $\mathrm{Li}, 2020)$, etc., forming a mainstream of medicine-related courses. There are few online teaching designs serving a specific course for English majors, let alone comprehensive discussions on oral English class.

Language is the carrier of culture and ideology, and language output is the main form of activity in oral English course. The flexibility of class organization and the diversity of teaching materials enable oral English teachers to dig out ideological and political elements in an all-around manner, spanning theoretical knowledge, value concepts and spiritual pursuits, etc., which, integrated into professional knowledge and skills, would have a subtle impact on students' thoughts and behaviors.

There is a long-standing paradox in oral English teaching. As a basic skill-oriented course for language majors, oral English attaches great importance to practicability and immediacy. However, relying on printed textbooks, most traditional oral English classes are conducted step by step in both content and structure based on the design of teaching materials. Since publication is time-sensitive, the topics that students are exposed to are, more often than not, outdated and marginalized, resulting in a poor "sense of acquisition" in oral English class. In addition, the majority of the textbooks for oral English currently used are compiled by European or American authors. It is undeniable that this guarantees the authenticity of language input. Nevertheless, a possible consequence is that students may become much more familiar with foreign figures, objects and cultural phenomena. When asked about their own country by foreigners in reality, however, they have little idea what and how to express, thus losing the initiative to promote excellent national culture and shape a favorable image of China, which violates the teaching objective and original intention of oral language classes. 
Online class is an inevitable choice for college teaching activities against the context of COVID-19 which itself serves as an ideal ideological and political resource for oral English. Therefore, with School of Foreign Studies, Guangzhou University of Chinese Medicine as an example, an exploration of online teaching design in the context of COVID-19 was carried out after an extraction of "ideological and political elements" in oral English class, in order to lend a reference to similar courses that seek for a stable, orderly and efficient online teaching mode. Based on the sophomore oral English class, the design and implementation of this course took as the teaching objects the four classes of English majors at Guangzhou University of Chinese Medicine, covering 108 students.

\section{Pre-Class Preparation}

\subsection{Selection of Tools and Platforms}

Undoubtedly, the rapid advancement of Internet technology and the popularization of smart phones and other mobile terminals facilitate the development of online teaching to a great extent. Meanwhile, it is foreseeable that, however, there would arise some common problems during online teaching in practice. First, the lack of standardized management in school makes it difficult to guarantee that students could actively engage themselves in online study in a self-disciplined way. Second, the unidirectional and usually "boring" lectures, instead of real-time interactions between the teacher and students, fail to catch students' attention, hence a decreased learning efficiency and motivation. Third, in the long run, online teaching may cause psychological problems for both teachers and students, how to deal with which would become an urgent issue. Fourth, compared with traditional class, online teaching is characterized with randomness. In other words, the freshness of the environment outshines students' thirst for knowledge. Some students take a delight in "bravoing" the anchor (that is, the teacher), or creating bullet screens. As a result, the entertainment-oriented approach tends to interfere with the normal teaching order. Last but not least, there is no standardized assessment system for online teaching. More than one student simply regards watching the teaching video the only task, thus bringing an obstacle for teachers to grasp students' degree of mastery (Li et al., 2020: 264).

In response to the above problems, with such features of oral English class taken into account as a relatively active atmosphere, and frequent and rapid word-of-mouth interactions, it's believed that the selected online teaching platform should meet the following conditions. First, it should give full play to the teacher as a supervisor and organizer while students are involved in discussions. Second, the convenience of voice connection should be prioritized in order to enhance the teacher-to-student and student-to-student oral interactions. Third, group and single games could be appropriately rewarded to the to offset the anxiety caused by the spatial barrier and the loss of in-class fun. Fourth, it should make possible multi-party and two-way simultaneous voice communication, creating face-to-face conversations as in the real world, rather than unidirectional and entertainment-oriented live broadcast teaching. Fifth, an embedded after-class management platform is needed on which students could upload and make comments on their homework.

After a general study and simulation operations of various resource platforms and teaching tools, it's found that the Rain Classroom fails to present the animation effects of the PowerPoint with content of one slide displayed at one time during the live broadcast. This on the one hand is not conducive to students' understanding of the content under explanation, and doesn't contribution to their concentration on the other (Zhang et al., 2020: 223). Equipped with a "Hands Up" function, the QQ Classroom allows students to be connected and heard after making a request to the teacher's end. This, however, is time-consuming and therefore is not suitable for oral English classroom where sometimes students need to race to answer questions or two or more people are required to talk at the same time. For some other teaching platforms, the discussion areas only support text messages, which cannot be used to send pictures or documents. After comparisons among the various tools covering iCourse, Rain Classroom, DingTalk, QQ Classroom, and Tencent series products in terms of advantages and disadvantages, as well as the scope of application, the DingTalk video conferencing is finally determined as the main method of online teaching. With the above platform selection principles considered, an online oral English teaching model featuring "DingTalk + WeChat Group + FiF" is formed.

\subsection{Introduction to the Platforms Used}

\subsubsection{DingTalk (Note 3)}

DingTalk is a smart mobile office platform produced by Alibaba. It provides clear and stable video conferencing for free, which supports 302 people online at the same time. Students can get access via smartphones, Pads, computers and other devices, and forward the current conference to the chat group, so that late students could enter the classroom at any time. The teacher as the initiator of the conference can record the whole lecture, which is convenient for students to review repeatedly after class. In addition, both the teacher and students can share 
their screens and files through either mobile phones or computers. Through the rich management functions such as "Mute All", "Remove Participants" and "Lock The Conference", etc., an orderly and controllable lecture by the teacher and presentations by students are possible throughout the whole process.

\subsubsection{WeChat (Group Voice Chat) (Note 4)}

Any member of the WeChat group can initiate an invitation for a voice chat, and others can join freely. The teacher joining each group in advance can come in and out at any moment without disturbing students. Taking turns among groups to monitor their discussions, the teacher can offer timely help to students. This, on the one hand, returns the initiative of conducting online oral activities to students, and on the other hand, realizes the teacher's supervisory role as in the traditional classroom.

\subsubsection{FiF Smart Teaching Platform (Note 5)}

Based on iFlytek's smart voice technology, the oral training system embedded in the FiF Smart Teaching Platform is a multi-terminal oral training and teaching management system, providing teachers and students with rich oral English practices and question types for assessment. Teachers could release tasks regularly on the platform, and thus easily have a clear picture of the progression and score distribution of a certain class, a certain student, or a certain task. Students can also log in and practice at any time according to their own language level and needs, and view their ranking in the system based on the amount of training. The scores students obtain from the system are used in this teaching design as a sub-module of formative evaluation.

\subsection{Preparation of Students}

Given the needs of pair and group discussions in oral English class, students are required to prepare two devices ("computer + smart phone" is the best, while two smart phones are also acceptable), so that one can be used to participate in the video conference teaching, and the other to read electronic teaching materials, $\log$ in WeChat, and initiate or join voice chats for group discussions at any time. With regard to the teaching platform, DingTalk (computer version and mobile version), WeChat, FiF oral training system (student version), etc. need to be downloaded in advance.

Before the formal class, students are required to attend an online trial, including the clarity of the screen, the fluency of the animation effects of the PowerPoint and the switching between the resources in the teacher's computer, the quality and volume of the teacher's and students' voices, and the delays of the audio and video clips, etc.

\section{Extraction of Ideological and Political Elements}

\subsection{Textbook Content}

The textbook used in the sophomore oral English course at School of Foreign Studies, Guangzhou University of Chinese Medicine is New Inside Out (the 2nd edition, Volume 4) published by Shanghai Foreign Language Education Press. Twelve units are included in the book, which are about Conversation, Taste, City, Story, Bargain, Mind, Digital, Law, Night, Footprints, Words and Conscience, respectively. In spite of a wide range, some subtopics chosen are unfamiliar to Chinese students and thus fail to arouse their interest, since the editors are all European and American teaching experts. As a consequence, parts of the topics are of little practical value because of a divorce from reality. Therefore, under the guidance of the IPTEC concept, some subtopics and in-class activities are replaced in this teaching design after an exploration of ideological and political elements, with the original general topics retained and the actual situation in China considered at well. For instance, for the topic "Taste" in the second unit, in addition to asking students to list the names, materials and flavors of the representative dishes of various countries as arranged in the textbook, it is also feasible to guide them to collect the general English expressions, cooking methods and ingredients of Chinese specialties before class. During this process, students could summarize the rules for Chinese cuisine translation, so that they can introduce to their classmates the flavors and even the legends or allusions of their hometown dishes in class. Another case in point is the topic "City" in Unit 3. Travel guides to four world-famous cities are given in the textbook, but none of them is for a Chinese city. In this teaching design, the teacher asks students to write a travel guide for their favorite Chinese city following the genre of those in the textbook before class. In class, role plays are carried out, in which tour guides and tourists would take turns to introduce and inquire about the city's famous scenic spots, local customs, history and culture.

Besides, in reference to the topic "Digital", students are required to focus on the recent development of China's electronic product industry, summarizing and reflecting on the opportunities and challenges in comparison with Western brands. For the topic "Footprints", a workable plan is to invite students to brainstorm their daily efforts to reduce carbon emissions, for the purpose of enhancing their sense of responsibility for protecting the global 
environment. As for the topic "Conscience", students' attention is drawn to the different attitudes between China and the West towards charity and the homelessness, so as to foster their virtues of integrity and kindness, and instill the value of respecting others and seeking common ground while reserving differences in a culturally diverse world.

In a nutshell, through the teacher's conscious mining of ideological and political elements out of the reasonable adjustment of the textbook content, students would be imperceptibly inspired to promote their familiar dietary habits, humanities, natural world, national industry, value system and the like during the process of accumulating vocabulary and phrases, and building language structures, hence a subtle cultivation of their pride in excellent Chinese culture and the establishment of a correct concept of value and outlook on life.

\subsection{Student Presentations}

In order to mobilize students' emotions and arouse their attention to class as soon as possible, the five to ten minutes before each class are used for student presentations. Since the stories of the medical care personnel that have emerged in the fight against the COVID-19 are particularly impressive for these students at a college of Chinese medicine, at the beginning of each oral class, the teacher arranges two students to tell a heroic deed respectively, talking about "the person you respect most in this epidemic". This can arouse students" esteem for medical staff, thus conducive to the establishment of a rational perspective on idols, life, and values, as well as the cultivation of patriotism, a broad vision, an awareness of civic responsibility, and the ability to distinguish right from wrong.

\subsection{Final Examination}

Centering around the COVID-19, the teacher prepares in advance a series of topics announced to the students 3 days before the final examination, which takes the form of oral narration within a limited time based on randomly-chosen questions. The topics include "What do you think of different countries' attitudes and actions towards the COVID-19?" "Supposing you were a recently returned overseas student, what would you do in the face of the quarantine?" "In addition to health threats, has the epidemic positively influenced your and your family's life?" "How do you think various countries could get out of the impact of the epidemic and restore their economies?" etc. A revelation of the topics in advance sets aside time for students to conduct targeted research, which enables them to form a positive attitude towards life during the in-depth thinking. Furthermore, it provides students an opportunity to reflect on the responsibilities of contemporary college students under the epidemic, thus creating their international vision featuring a community with shared future for mankind.

\section{Teaching Process Design}

- DingTalk training groups are established for each class before the start of the online teaching.

- As the very beginning, a simple click on "Video Call" makes it possible for the teacher to initiate a video conference, inviting all members of the group to join the class. While students are entering the classroom, switches can be made by means of such buttons as "Mute", "Camera On", "Mute All", etc. (Figure 2).

- The teacher could, after all the students join the class, display the content in his/her device with the function "Share Screen". Moreover, a click on "Grid" presents a name list of the students whose microphones are in the charge of the teacher, thus facilitating the voice interaction between the teacher and one or more students (Figure 2).

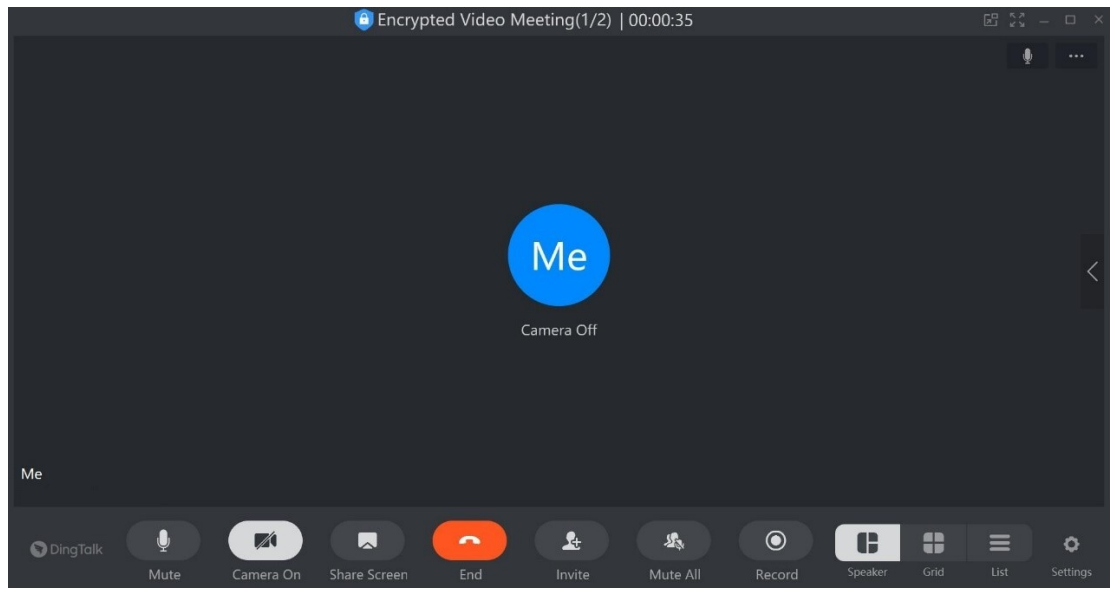

Figure 2. Interface of DingTalk online classroom (with the teacher in only) 
- While students are listening to the lecture in the video conference using the computer, they can also post text messages or pictures in real time in the DingTalk group through the mobile phone, which plays the role of a bullet screen. This, on the one hand, allows students to pose questions in a non-voice manner and submit in-class tasks (Figure 3), and, on the other, enables the teacher by inputting text at any time to explain difficult and puzzling points (Figure 4).

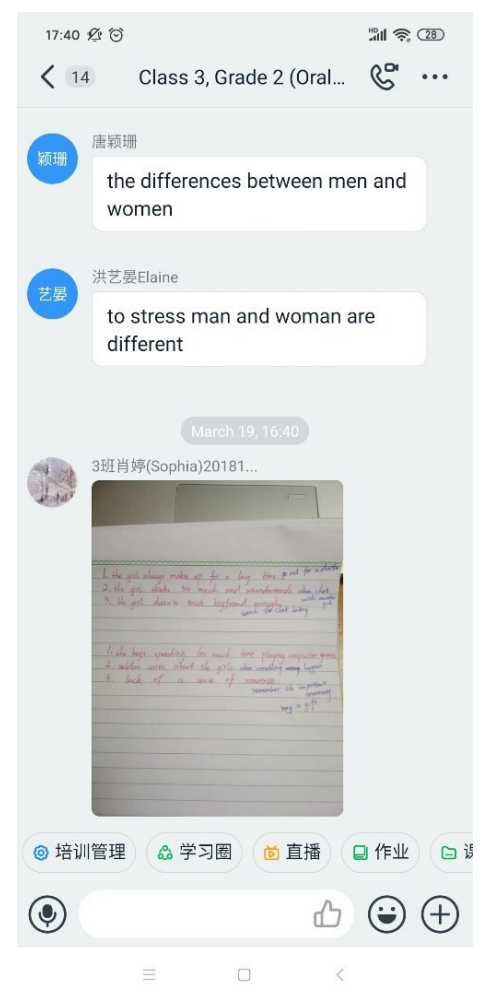

Figure 3. Students' report on group discussion results

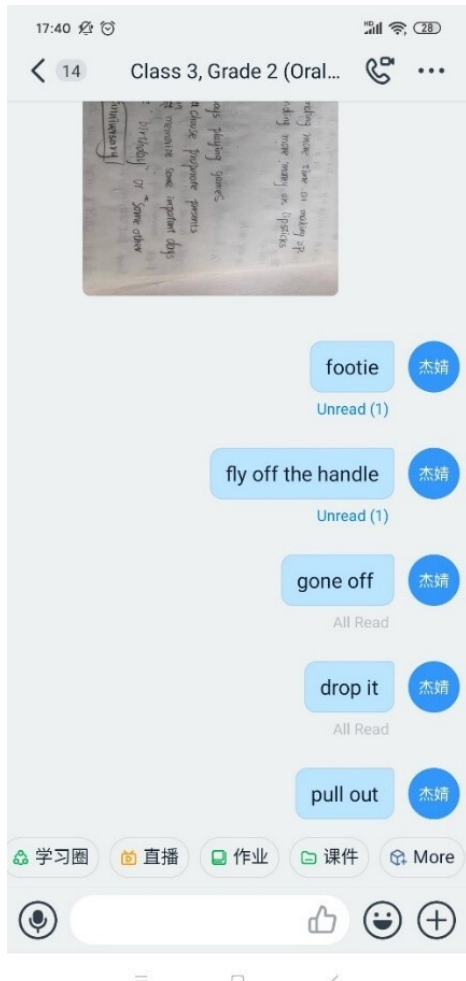

Figure 4. Teacher's reply 
- In addition, students can also make presentations as the keynote speaker by sharing their screens, with such functions available as synchronized PowerPoint display, voice explanation, audio and video playing, classmate invitation, and one-to-one interaction with the teacher, etc.

\section{After-Class Management}

In the homework management module of DingTalk group, the teacher can set the deadline, time span when the assignment is open to public, submission method and other parameters. Data concerning the completion rate of and comments by either the teacher or students on a certain assignment can be clearly shown on the homework interface (Figure $5 \& 6$ ). In addition, a "DING" function is available as a "strong reminder" for students who haven't completed their homework before the deadline.

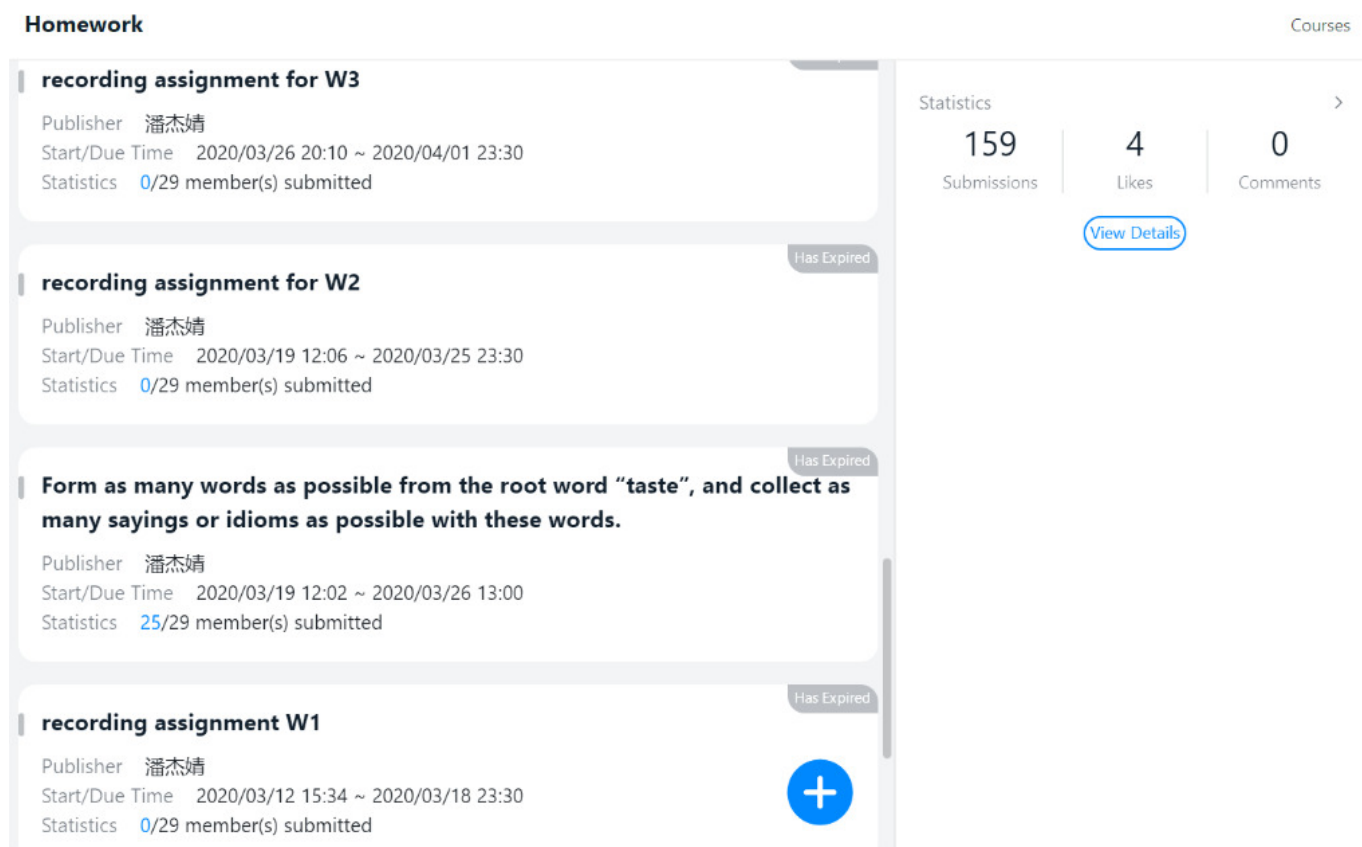

Figure 5. Interface of DingTalk homework release

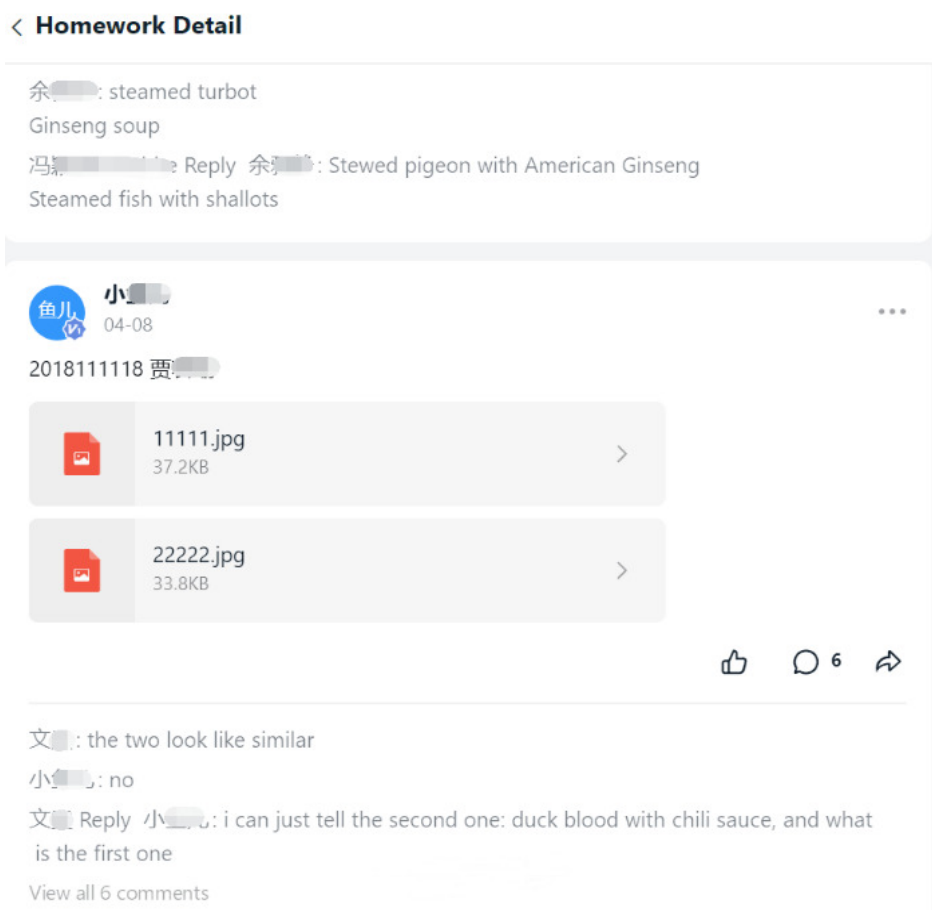

Figure 6. Students' active replies on each other's homework 


\section{Concluding Remarks}

With the sophomore oral English course of School of Foreign Studies, Guangzhou University of Chinese Medicine as a case, an online teaching mode of oral English featuring "DingTalk + WeChat Group + FiF" is proposed based on a full exploration of "ideological and political elements". As far as its content is concerned, this teaching design is permeated with ideological and political elements in all links, hence a combination of explicit and implicit educations. On top of that, it's also characterized with the following features.

In terms of voice interaction, teacher-to-student and student-to-student communications are supported in a multi-party manner at any time. The selection of the DingTalk video conference over the traditional mode of one-way live broadcast fulfill the requirement of high-frequency interactions in oral English class. Turning on the microphone, students could take the initiative to speak at any time, which saves the time otherwise wasted in "requesting and queuing to be connected", hence an improvement of classroom efficiency.

Besides, a complex is created where one online classroom is systematically nested in another among the various platforms. Considering the requirement of the organizational form of oral English course, each class is divided into several teams with their respective WeChat groups built. Group discussions can then be carried out through WeChat voice group chat, which acts as a "second online classroom" embedded in the DingTalk online classroom. On the one hand, the teacher can supervise the activities and provide help therein without any interruption. And on the other hand, with collaborative study among students, the traditional classroom is restored to the largest extent, which is of great help to alleviate their anxiety caused by somewhat "dull" online teaching.

Occasionally, group and single games would be arranged, which enrich the organization of the classroom. Students' performance in group games is in most cases influenced by the effect of their preview work. For each class, one winning team will be determined, which is appropriately rewarded by the teacher. With regard to the single game, FiF oral training system will provide a ranking based on students' self-study. Students have shown great enthusiasm in the group and single games, making up for the part of learning interest filtered out by technical barriers.

Last but not least, this teaching design provides private and convenient classroom and homework management. It is under the premise of independent and exclusive DingTalk and WeChat groups that the participation in video conferences, text and picture exchanges and the release and upload of tasks are conducted, which guarantees the privacy of each teaching link and thus improves students' degree of involvement.

\section{Acknowledgments}

Our special gratitude goes to the editors and anonymous reviewers of English Language Teaching for their valuable insights and constructive suggestions.

\section{References}

Cui, Y. H., \& Che, R. S. (2020). Reflections on the ideology and politics of online courses. Review of High Education, 3, 81-85.

Fu, H. Y., Yang, W., Yuan, H. Y., Yan, D. M., Ni, W. H., \& Qi, Y. (2020). Online teaching of "Medical Immunology" that integrates Knowledge-Ability-Values cultivation in "Anti-epidemic". Chinese Journal of Immunology, 36(18), 2229-2231. https://doi.org/10.3969/j.issn.1000-484X.2020.18.015

Han, Q. J., Shi, T., Hao, G. X., \& Zhang, J. (2020). Ideological and political education in online teaching. Chinese Journal of Immunology, 36(18), 2271-2274. https://doi.org/10.3969/j.issn.1000-484X.2020.18.027

Jiang, P. T., Guo, N., Gao, X. C., Wang, N., \& Jiang, F. L. (2020). Online teaching design and practice of Medical Immunology for disciplines of humanities and social sciences in medical university. Chinese Journal of Immunology, 36(19), 2339-2342. https://doi.org/10.3969 /j.issn.1000-484X.2020.19.010

Jin, L. M., Hou, X. Y., Men, L., Xu, Y. B., \& M, J. L. (2020). On teaching strategies of Pharmaceutical Analysis against COVID-19. Light Industry Science and Technology, 36(8), 197-198.

Li, F. F. (2020). Exploring ideological and political construction of online teaching in universities. Heilongjiang Education (Higher Education Research \& Appraisal), 9, $42-43$.

Li, K. H., Liu, Y., Xie, H. X., Wang, L., Zhang, L. L., \& Luo, E. (2020). Discussion on online teaching mode under the COVID-19 epidemic. China Medical Education Technology, 34(3), 264-266. https://doi.org/10.13566/j.cnki.cmet.cn61-1317/g4.202003003 
Sun, B. (2020). An exploration of ideological and political elements in foreign languages education. China Journal of Multimedia \& Network Teaching, 6, 188-191.

Wang, X. J., \& Shi, Y. (2020). On the ideological and political education of college courses in the new era. Journal of Xinjiang Normal University (Philosophy and Social Sciences), 41(2), 50-58. https://doi.org/10.14100/j.cnki.65-1039/g4.20191125.001

Xi, J. P. (2016). Xi Jinping emphasized at the National Conference on Ideological and Political Work in Colleges and Universities: Conduct ideological and political work throughout the entire process of education and teaching to create a new situation in the development of China's higher education. People's Daily.

Xu, G. H., Bai, Y. M., Huang, F., Yan, S. X., Sun, R., Wang, A. H., ... Kang, J. (2020). Online teaching reform and practice of "San Quan Education System" for nursing. Chinese Nursing Management, 20(7), 975-978. https://doi.org/10.3969/j.issn.1672-1756.2020.07.004

Zhang, L., Liu, J., Sun, B., Huo, F. Q., Guo, Y., Chen, L., ... Yan, J. Q. (2020). Exploration and Reflection on Physiology Online Teaching Mode under the Novel Coronavirus (2019-nCoV) Epidemic. Medical Education Research and Practice, 28(2), 221-224. https://doi.org/10.13555/j.cnki.c.m.e.2020.02.011

\section{Notes}

Note 1. Available at: https://www.moe.gov.cn/jyb_xwfb/gzdt_gzdt/s5987/202001/t20200127_416672.html, 2020-01-27

Note 2. Available at: https://www.moe.gov.cn/jyb_xwfb/gzdt_gzdt/s5987/202001/t20200129_416993.html, 2020-01-29

Note 3. Available at: https://www.dingtalk.com/?lwfrom=2017120202091367000000111\&source=1001

Note 4. Available at: https://weixin.qq.com/

Note 5. Available at: https://www.fifedu.com/iplat/html/index.html

\section{Copyrights}

Copyright for this article is retained by the author(s), with first publication rights granted to the journal.

This is an open-access article distributed under the terms and conditions of the Creative Commons Attribution license (http://creativecommons.org/licenses/by/4.0/). 